\title{
Adding calcium scoring to myocardial perfusion imaging: Does it alter physicians' therapeutic decision making?
}

\author{
Ron Blankstein, MD, and Sharmila Dorbala, MD
}

\section{See related article, pp. 188-196}

Tests do not directly affect clinical status. Instead we must presume that they lead clinicians and patients to modify behavior, which hopefully will lead to fewer clinical events.

-Report of a workshop sponsored by the National Heart, Lung, and Blood Institute ${ }^{1}$

While the paucity of studies relating cardiovascular imaging to patient outcomes has drawn much attention, the complex nature of cardiovascular disease makes it extremely hard to study how a test performed today can lead to better outcomes in the future. Realizing this, a simpler question is whether imaging testing leads to changes in patient or physician behavior. In other words, does the test lead to initiation of new therapies that would not have otherwise been considered?

Among symptomatic patients with known or suspected coronary artery disease (CAD), myocardial perfusion imaging (MPI) provides important information about the presence and extent of ischemia or infarction and can be used to predict the presence of hemodynamically significant obstructive CAD. This information can then be used in deciding between medical therapies and revascularization. Positron emission tomography (PET) is a superb technique to assess myocardial perfusion. ${ }^{2}$ While PET has many advantages over SPECT techniques, such as improved image resolution, robust attenuation correction and ability to

From the Noninvasive Cardiovascular Imaging Program, Departments of Medicine (Cardiovascular Division) and Radiology, Brigham and Women's Hospital, Harvard Medical School, Boston, MA.

Reprint requests: Sharmila Dorbala, MD, Noninvasive Cardiovascular Imaging Program, Departments of Medicine (Cardiovascular Division) and Radiology, Brigham and Women's Hospital, Harvard Medical School, 70 Francis Street, Boston, MA 02115; sdorbala@ partners.org.

J Nucl Cardiol 2010;17:168-71.

$1071-3581 / \$ 34.00$

Copyright (C) 2009 by the American Society of Nuclear Cardiology. doi:10.1007/s12350-009-9185-7 measure absolute myocardial blood flow, it is limited in its ability to identify pre-clinical atherosclerosis and severe multi-vessel atherosclerosis (balanced ischemia). On the other hand, coronary artery calcium (CAC) scoring identifies whether coronary calcifications are present or absent, and reliably quantifies the burden of calcified coronary atherosclerosis (though not necessarily representing obstructive CAD). ${ }^{3}$ The exam is simple, does not require intravenous contrast and can be performed with a relatively low radiation dose $(\sim 1-2 \mathrm{mSv})^{4}$ on the majority of CT scanners as well as on hybrid SPECT/CT and PET/CT platforms.

In patients undergoing MPI, CAC scoring can be used to better define the presence or absence of calcified atherosclerosis, thereby resulting in improved risk assessment. In highlighting the complementary information provided by these tests, several recent studies have showed that approximately $21-47 \%$ of patients with normal MPI have extensive coronary calcifications (i.e. CAC > 400) ${ }^{5-8}$ Among patients who are otherwise considered low to intermediate risk, such findings could be used to reclassify their risk, thereby indicating that more aggressive therapies should be considered.

The increased availability of hybrid SPECT/CT and PET/CT platforms has facilitated the acquisition of CAC score together with MPI during a single setting. One of the arguments for such an approach is that the identification of CAC can be used to identify the presence and extent of atherosclerosis. On the other hand, when extensive calcifications are found, perfusion imaging can be used to determine the physiologic significance of plaque. ${ }^{9}$ However, in contrast to screening cohorts undergoing CAC scoring, patient cohorts undergoing combined MPI and CAC at the same setting have a higher pretest likelihood of CAD as they are more likely to be symptomatic patients referred for an evaluation of ischemia. ${ }^{6,7}$ Patients referred for PET MPI may have an even higher risk, due to their reduced functional capacity and/or presence of obesity. However, even in such patient populations, the presence of severe calcified coronary atherosclerosis provides substantial incremental prognostic value. ${ }^{7}$ Therefore, the logical next question is whether the improved diagnosis of coronary atherosclerosis by CAC score leads to changes in 
physician treatment strategies (initiate or optimize medical therapy) or patient behavior (lifestyle changes)? A corollary to this question is whether aggressive management of risk factors based on CAC score averts future adverse cardiovascular events.

Three prior studies attempted to address the first question. $^{10-12}$ Wong and colleagues ${ }^{11}$ showed in 703 asymptomatic patients that underwent CAC score, that the presence of CAC was an independent predictor of risk reducing behaviors such as increased use of aspirin and cholesterol lowering medications as well as consultation with a physician. In a separate study, Taylor et $\mathrm{al}^{10}$ attempted to answer the question of whether a screening calcium score results in changes in subsequent aspirin and statin. Among 1640 active duty male army personnel, they showed that the presence of coronary calcification $(\mathrm{CAC}>0)$ was associated with an independent 3-fold greater likelihood of statin and aspirin usage. Thompson et $\mathrm{al}^{12}$ previously studied 200 consecutive patients who were referred for CAC scoring following a negative MPI. Upon subsequent follow-up, the $17.5 \%$ of patients with a CAC score greater than 100 had a significantly greater use of aspirin and lipid lowering therapies.

However, data demonstrating that the use of imaging based markers such as CAC leads to reduced future cardiovascular events is limited. This is due to the fact that studies addressing this question would be complex and expensive as a long follow-up period would be required. Moreover, randomization of patients with risk factors such as CAC into different types of therapy (i.e. with no therapy representing the most extreme option) may be limited due to ethical considerations. The St. Francis Heart Study ${ }^{13}$ tested the hypothesis that treatment with atorvastatin and vitamins $\mathrm{C}$ and $\mathrm{E}$ would reduce a composite of all atherosclerotic cardiovascular disease events in a population with elevated CAC. The study results were negative for the primary endpoint, but showed a trend toward lower events in subgroup of patients with CAC score of $>400$ in the treatment group compared to the control group. The authors concluded that the study failed to detect differences in outcomes between the two treatment groups, likely because of inclusion of lower risk subjects or an inappropriate selection of the treatment threshold of CAC score.

The study by Dr Bybee and colleagues, published in this issue of JNC, represents an important addition to the emerging literature regarding the integration of MPI and calcium scoring. The authors sought to determine whether the identification of coronary atherosclerosis among patients presenting for a clinical PET scan is associated with subsequent changes to medical therapies. The 30-day rate of initiation or optimization of dose of medical therapies (statins, aspirin, beta-blockers, ACE inhibitors, and omega-3 fatty acids) was determined by chart review. Bybee et al studied 760 patients with no prior CAD and a normal clinically indicated vasodilator stress MPI study. In this cohort, $64.1 \%$ had coronary calcifications with $30 \%$ having CACS $\geq 100$ and $14 \%$ having $\mathrm{CACS} \geq 400$.

In regards to medical therapies, several important findings are noteworthy. First, at baseline, a significant proportion of patients who were found to have extensive coronary calcifications (i.e. CAC score of >400) were not treated with statin therapy (52\%) or aspirin (49\%). Within 30 days of the PET/CT exam, $43 \%$ of the study cohort had initiation or augmentation of medical therapies. Not surprisingly, patients with a CAC score $>400$ were almost twice as likely to have an increase in medical therapies, a finding that was predominantly driven by increased use of statin medications. It might have been interesting to find out what is the CAC score threshold that triggered changes in therapy although such an analysis (ROC curve) would likely require a larger cohort.

Perhaps somewhat less expected was the finding that initiation of aspirin therapy (12\% of the cohort) did not differ between patients with or without calcifications. Also of note is that $31 \%$ of patients with CAC $=0$ had a change in therapies following the PET/CT scan, suggesting that changes in therapy were used for risk factor reduction (i.e. treatment of dyslipidemia or hypertension) rather than for secondary prevention of ischemic heart disease. In a multi-variable model, increased CAC emerged as an independent predictor of initiation or optimization of medical therapies and for every 100 unit increase in Agatston score, the odds ratio for predicting changes in medical therapies was fairly modest, yet statistically significant $(\mathrm{OR}=1.05 ; P=.003)$. Also, $18 \%$ of the patients in the high Framingham risk score group had no coronary artery calcium, supporting the concept that the current risk prediction models may over estimate risk in certain cohorts (e.g. diabetics ${ }^{14}$ ) and that there may be a role for CAC to further refine riskassessment in selected cohorts. ${ }^{15}$

The overall results of this study are complementary to existing literature and support the notion that the CAC score may influence physician practices and lead to optimization of medical therapy in patients referred for a stress PET study. An important strength of the study by Bybee and colleagues is that simultaneous assessment of MPI and CAC was performed on all consecutive patients who did not have known CAD. Thus, the study is not limited by referral bias and the results are applicable to similar populations of patient presenting for MPI.

Despite these attributes, the findings from this study must be interpreted in the context of the study design and the cohort studied. First, the observed changes in 
therapy were driven by physician judgment. Consequently, when compared to recommended guidelines and as is often the case in the real-world, some patients were over- or undertreated. Nevertheless, assessing the impact of test results on physician behavior is important and is ultimately one of the most relevant metrics that determines patient outcomes. Second, since patients in this study were referred for pharmacologic MPI, they represent a higher risk cohort (for instance, $22.1 \%$ had diabetes and reportedly approximately $40 \%$ were expected to have CAD based on the Diamond and Forrester criteria). Thus, the results of this study cannot be directly extrapolated to lower risk populations. In addition, due to the higher risk of this cohort, one may expect that in some of the patients studies, aggressive medical therapies would be recommended regardless of CAC. In such patients, the added value of CAC scoring may be lower. On the other hand, in patients in whom risk reclassification would result by the addition of $\mathrm{CAC}$, the value of a combined exam would be higher. When considering the impact of the test on risk reclassification, it is noteworthy that in the study by Bybee et al, approximately $18 \%$ of the patients in the high FRS group had no calcium.

Another consideration is that the medical therapies considered in this study included not only aspirin and statins, but also agents used in the treatment of heart failure and hypertension. This suggests that some of the changes in therapies were instituted for reasons other than treatment of ischemic heart disease (e.g. hypertension or heart failure). To address this point, the authors showed that there was no significant difference in the mean ejection fraction between those with and without change in medical therapy, yet in the multivariate model a lower EF was one of only two independent predictors (the other being CAC) for predicting changes in medical therapy.

Lastly, although not the emphasis of this study, the methods for CAC scan acquisition used in this study provide an important reminder as to the importance of using appropriate scanner settings to minimize patient radiation dose. Among scans performed on the 64-slice systems, axial acquisition using prospective ECG gating was utilized $(120 \mathrm{kV}, 100 \mathrm{~mA}, 0.6 \mathrm{~mm}$ collimation) and the resulting effective dose was estimated at $1-2 \mathrm{mSv}$, which could have further been decreased by using thicker collimation of 2.5 to $3 \mathrm{~mm}$. On the other hand, for scans utilizing the 16-slice system, a helical acquisition with tube current modulation was used $(120 \mathrm{kV}$, $200 \mathrm{mAs}, 1.5 \mathrm{~mm}$ collimation, 0.28 pitch) and the estimated radiation dose for these scans was considerably higher (3-5 mSv). When suboptimal techniques (i.e. helical acquisition) are used for CAC scoring, ${ }^{16}$ doses that are significantly higher than projected may result. This emphasizes the need to use the optimal CAC scanning protocol to minimize radiation dose.

In conclusion, the study by Bybee and colleagues represents one of the first studies assessing the impact of hybrid PET MPI and CAC scoring on physician prescription practices. By showing that among patients with normal MPI, the presence of CAC has a significant impact on physician prescribing patterns, they have contributed to the evidence supporting the selective use of CAC among patients referred for PET MPI. While this study provides a rationale for a selective use of combined CAC and MPI imaging, other considerations such as cost and availability of hybrid scanners will be important factors to consider when establishing recommendations for the adoption of hybrid imaging strategies. Importantly, answering questions on the added value of CAC score to nonischemic MPI will also require comparing how different patient cohorts and different imaging modalities (i.e. exercise treadmill testing, CT angiography) affect medical therapies. Ongoing studies such as the SPARC study will further inform such questions. Ultimately, however, it will be incumbent upon our field to show that imaging testing translates into improved patient outcomes and are costeffective.

\section{Acknowledgment}

This work was funded by an NIH grant (\#1 K23HL09229901A2).

\section{References}

1. Douglas PS, Taylor A, Bild D, et al. Outcomes research in cardiovascular imaging: Report of a workshop sponsored by the National Heart, Lung, and Blood Institute. JACC Cardiovasc Imaging 2009;2:897-907.

2. Bengel FM, Higuchi T, Javadi MS, et al. Cardiac positron emission tomography. J Am Coll Cardiol 2009;54:1-15.

3. Agatston AS, Janowitz WR, Hildner FJ, et al. Quantification of coronary artery calcium using ultrafast computed tomography. J Am Coll Cardiol 1990;15:827-32.

4. Gerber TC, Carr JJ, Arai AE, et al. Ionizing radiation in cardiac imaging: A science advisory from the American Heart Association Committee on Cardiac Imaging of the Council on Clinical Cardiology and Committee on Cardiovascular Imaging and Intervention of the Council on Cardiovascular Radiology and Intervention. Circulation 2009;119:1056-65.

5. Berman DS, Wong ND, Gransar H, et al. Relationship between stress-induced myocardial ischemia and atherosclerosis measured by coronary calcium tomography. J Am Coll Cardiol 2004;44:923-30.

6. Chang SM, Nabi F, Xu J, et al. The coronary artery calcium score and stress myocardial perfusion imaging provide independent and complementary prediction of cardiac risk. J Am Coll Cardiol 2009;54:1872-82. 
7. Schenker MP, Dorbala S, Hong EC, et al. Interrelation of coronary calcification, myocardial ischemia, and outcomes in patients with intermediate likelihood of coronary artery disease: A combined positron emission tomography/computed tomography study. Circulation 2008;117:1693-700.

8. Uebleis C, Becker A, Griesshammer I, et al. Stable coronary artery disease: Prognostic value of myocardial perfusion SPECT in relation to coronary calcium scoring-long-term follow-up. Radiology 2009;252:682-90.

9. Schepis T, Gaemperli O, Koepfli P, et al. Added value of coronary artery calcium score as an adjunct to gated SPECT for the evaluation of coronary artery disease in an intermediate-risk population. J Nucl Med 2007;48:1424-30.

10. Taylor AJ, Bindeman J, Feuerstein I, et al. Community-based provision of statin and aspirin after the detection of coronary artery calcium within a community-based screening cohort. J Am Coll Cardiol 2008;51:1337-41.

11. Wong ND, Detrano RC, Diamond G, et al. Does coronary artery screening by electron beam computed tomography motivate potentially beneficial lifestyle behaviors? Am J Cardiol 1996;78:1220-3.

12. Thompson RC, McGhie AI, Moser KW, et al. Clinical utility of coronary calcium scoring after nonischemic myocardial perfusion imaging. J Nucl Cardiol 2005;12:392-400.

13. Arad Y, Spadaro LA, Roth M, et al. Treatment of asymptomatic adults with elevated coronary calcium scores with atorvastatin, vitamin C, and vitamin E: The St. Francis Heart Study randomized clinical trial. J Am Coll Cardiol 2005;46:166-72.

14. Simmons RK, Coleman RL, Price HC, et al. Performance of the UK Prospective Diabetes Study risk engine and the Framingham risk equations in estimating cardiovascular disease in the EPICNorfolk cohort. Diabetes Care 2009;32(4):708-13.

15. Bax JJ, Schuijf JD. Can coronary calcification define the warranty period of a normal myocardial perfusion study? Mayo Clin Proc 2008;83:10-2.

16. Kim KP, Einstein AJ, Berrington de Gonzalez A. Coronary artery calcification screening: Estimated radiation dose and cancer risk. Arch Intern Med 2009;169:1188-94. 